
\title{
$\begin{array}{ll}\text { Research Square } & \begin{array}{l}\text { Preprints are preliminary reports that have not undergone peer review. } \\ \text { They should not be considered conclusive, used to inform clinical practice, } \\ \text { or referenced by the media as validated information. }\end{array}\end{array}$ \\ Expectation Identity of the Hypergeometric Distribution and Its Application in the Calculations of High-Order Origin Moments
}

\section{Yuan-Quan Wang}

Chongqing University

Ying-Ying Zhang ( $\sim$ robertzhangyying@qq.com )

Chongqing University

Jia-Lei Liu

Chongqing University

\section{Research Article}

Keywords: expectation identity, high-order origin moments, hypergeometric distribution, mathematical induction, Stirling numbers of the second kind

Posted Date: December 20th, 2021

DOI: https://doi.org/10.21203/rs.3.rs-1180214/v1

License: (c) (i) This work is licensed under a Creative Commons Attribution 4.0 International License. Read Full License

Version of Record: A version of this preprint was published at Communications in Statistics - Theory and Methods on January 4th, 2022. See the published version at https://doi.org/10.1080/03610926.2021.2024235. 


\title{
Expectation Identity of the Hypergeometric Distribution and Its Application in the Calculations of High-Order Origin Moments
}

\author{
Yuan-Quan Wang ${ }^{\mathrm{a}}$, Ying-Ying Zhang, ${ }^{\mathrm{a}, \mathrm{b}, *}$, Jia-Lei Liu ${ }^{\mathrm{a}}$ \\ ${ }^{a}$ Department of Statistics and Actuarial Science, College of Mathematics and Statistics, \\ Chongqing University, Chongqing, China \\ ${ }^{b}$ Chongqing Key Laboratory of Analytic Mathematics and Applications, Chongqing \\ University, Chongqing, China
}

\begin{abstract}
We provide a novel method to analytically calculate the high-order origin moments of a hypergeometric distribution, that is, the expectation identity method. First, the expectation identity of the hypergeometric distribution is discovered and summarized in a theorem. After that, we analytically calculate the first four origin moments of the hypergeometric distribution by using the expectation identity. Furthermore, we analytically calculate the general $k$ th $(k=1,2, \ldots)$ origin moment of the hypergeometric distribution by using the expectation identity, and the results are summarized in a theorem. Moreover, we use the general $k$ th origin moment to validate the first four origin moments of the hypergeometric distribution. Next, the coefficients of the first ten origin moments of the hypergeometric distribution are summarized in a table containing Stirling numbers of the second kind. Moreover, the general $k$ th origin moment of the hypergeometric distribution by using the expectation identity is restated by another theorem involving Stirling numbers of the second kind. Finally, we provide some numerical and theoretical results.
\end{abstract}

Keywords: expectation identity, high-order origin moments, hypergeometric

\footnotetext{
* Corresponding author

Email addresses: 335876119@qq.com (Yuan-Quan Wang), robertzhangyying@qq.com or robertzhang@cqu.edu.cn (Ying-Ying Zhang), 1418812610@qq.com (Jia-Lei Liu)

URL: https://zhangyingying319. wordpress .com (Ying-Ying Zhang)

Yuan-Quan Wang and Ying-Ying Zhang are co-first authors.
}

Preprint submitted to Communications in Statistics-Theory and Methods December 17, 2021 
distribution, mathematical induction, Stirling numbers of the second kind 2020 MSC: 62Exx, 62Qxx, 05A10

\section{Introduction}

The hypergeometric distribution has attracted continuous interest in the literature since 2000. Childs and Balakrishnan (2000) examined some approximations to the multivariate hypergeometric distribution with applications to hypothesis testing. Kumar (2002) introduced extended generalized hypergeometric probability distributions. Hida and Akahira (2003) considered an improvement on the approximation to the generalized hypergeometric distribution. Dinwoodie et al. (2004) presented two new transform methods for computing with hypergeometric distributions on lattice points. Hush and Scovel (2005) provided an improved concentration of measure theorem for the hypergeometric distribution. Kumar (2007) studied some properties of bivariate generalized hypergeometric probability distributions. Lahiri et al. (2007) investigated normal approximation to the hypergeometric distribution in nonstandard cases and established a sub-Gaussian Berry-Esseen theorem. Fog (2008) developed sampling methods for Wallenius' and Fisher's noncentral hypergeometric distributions. Li and Tian (2010) provided a simple algorithm for the high-order origin moments of the hypergeometric distribution. Eisinga and Pelzer (2011) proposed saddlepoint approximations to the mean and variance of the extended hypergeometric distribution. Lebrun (2013) proposed an efficient time/space algorithm to compute rectangular probabilities of multinomial, multivariate hypergeometric and multivariate Plya distributions. Liu and Wang (2014) obtained the high-order origin moments, the high-order central moments, and the high-order cumulants of the hypergeometric distribution. de Klerk et al. (2015) considered an error analysis for polynomial optimization over the simplex based on the multivariate hypergeometric distribution. Danielian et al. (2016) proposed a new regularly varying generalized hypergeometric distribution of the second type. Greene and Wellner (2017) established exponential bounds for the hypergeometric distri- 
bution. Mano (2017) discussed partition structure and the A-hypergeometric distribution associated with the rational normal curve. Takayama et al. (2018) considered A-hypergeometric distributions and Newton polytopes. Hafid et al. (2020) proposed a novel methodology-based joint hypergeometric distribution to analyze the security of sharded blockchains. Krishnamoorthy and Lv (2020) constructed prediction intervals for hypergeometric distributions. Themangani et al. (2020) introduced a generalized hypergeometric distribution and discussed its applications on univalent functions. For earlier literature on the hypergeometric distribution, we refer readers to Riordan (1937); Kemp and Kemp (1956); Lieberman and Owen (1962); Harkness (1965); Brunk et al. (1968); Ord (1968); Skibinsky (1970); Molenaar (1973); Tripathi and Gurland (1977); Guenther (1978); Chvatal (1979); Lai (1979); Sibuya and Shimizu (1981); Panaretos (1983); Ling and Pratt (1984); Kachitvichyanukul and Schmeiser (1988); Kochman et al. (1989); Tohma et al. (1991); Wu (1993); Lin and Hsiung (1994); Lin et al. (1994); Ma (1999).

Exponential families include the continuous families (normal, gamma, and beta) and the discrete families (binomial, Poisson, and negative binomial). For the continuous exponential families, there are some expectation identities that rely on integration by parts. Stein's Lemma (Lemma 3.6.5 in Casella and Berger (2002)) gives an expectation identity for the normal family. Moreover, the expectation identities of the gamma and beta families are given in Exercise 3.49 of Casella and Berger (2002). It is worth noting that these expectation identities are useful in the calculations of high-order origin moments of the corresponding families. The discrete analogs of the expectation identities of the continuous exponential families are given in Theorem 3.6.8 of Casella and Berger (2002), which gives the expectation identities for the Poisson and negative binomial families (see also Hwang (1982)). Note that the Poisson and negative binomial families take countable infinite values. For the binomial family taking finite values, similar to the deviations of the Poisson and negative binomial expectation identities, Zhang et al. (2019) discovered an expectation identity for the binomial family and obtained a closed-form formula of the high-order origin moments 
for the binomial family by using the binomial expectation identity.

The analytical calculations of the high-order origin moments of the hypergeometric distribution are quite challenging. In this paper, we provide a novel method to analytically calculate the high-order origin moments of the hypergeometric distribution, that is, the expectation identity method. First, the expectation identity of the hypergeometric distribution is discovered and summarized in a theorem. After that, we analytically calculate the first four origin moments and the general $k$ th $(k=1,2, \ldots)$ origin moment of the hypergeometric distribution by the expectation identity method. Moreover, we use the general $k$ th origin moment to validate the first four origin moments of the hypergeometric distribution. Finally, the coefficients of the first 10 origin moments of the hypergeometric distribution are shown in a table. By comparing the tables in the literature, we find that the coefficients are Stirling numbers of the second kind.

The rest of the paper is organized as follows. In the next Section 2, we discover an expectation identity of the hypergeometric distribution. In Section 3 , we will use the expectation identity of the hypergeometric distribution to calculate its high-order origin moments. First, we will use the expectation identity of the hypergeometric distribution to calculate its first four origin moments. Second, we will use the expectation identity of the hypergeometric distribution to calculate its general $k$ th $(k=1,2, \ldots)$ origin moment. Third, we will use the general $k$ th origin moment to validate the first four origin moments of the hypergeometric distribution. Finally, the coefficients of the first 10 origin moments of the hypergeometric distribution are summarized in a table. In Section 4 , we will provide some numerical and theoretical results. Some conclusions and discussions are provided in Section 5.

\section{Expectation Identity of the Hypergeometric Distribution}

In this section, we discover an expectation identity of the hypergeometric distribution. The expectation identity is useful in analytical calculations of the high-order origin moments of the hypergeometric distribution. 
Let $Y \sim H G(N, M, n)$ have a hypergeometric distribution with probability mass function (pmf) given by (see Casella and Berger (2002))

$$
P(Y=y \mid N, M, n)=\frac{\left(\begin{array}{c}
M \\
y
\end{array}\right)\left(\begin{array}{c}
N-M \\
n-y
\end{array}\right)}{\left(\begin{array}{c}
N \\
n
\end{array}\right)}, y=0,1, \ldots, n,
$$

where $N$ is the total number of identical balls in a large urn, $M$ is the number of red balls in the urn, $N-M$ is the number of green balls in the urn, $n$ is the total number of selected balls, $y$ is the number of selected balls that are red, and $n-y$ is the number of selected balls that are green. Similarly, let $X \sim H G(N-1, M-1, n-1)$ have a hypergeometric distribution with pmf given by

$$
P(X=x \mid N-1, M-1, n-1)=\frac{\left(\begin{array}{c}
M-1 \\
x
\end{array}\right)\left(\begin{array}{c}
N-M \\
n-1-x
\end{array}\right)}{\left(\begin{array}{c}
N-1 \\
n-1
\end{array}\right)}, \quad x=0,1, \ldots, n-1 .
$$

By definition,

$$
\mathrm{E} Y^{k}=\sum_{y=0}^{n} y^{k} \frac{\left(\begin{array}{c}
M \\
y
\end{array}\right)\left(\begin{array}{c}
N-M \\
n-y
\end{array}\right)}{\left(\begin{array}{l}
N \\
n
\end{array}\right)}
$$

for $k=0,1,2, \ldots$

The expectation identity of the hypergeometric distribution is discovered and summarized in the following theorem whose proof is simple as with many important results.

Theorem 1. Let $X \sim H G(N-1, M-1, n-1)$ and $Y \sim H G(N, M, n)$. Moreover, let $g(x)$ be a function satisfying $-\infty<\mathrm{E}[g(X)]<\infty$ and $-\infty<g(-1)<$ $\infty$. Then we have the following expectation identity of the hypergeometric distribution:

$$
\mathrm{E}[g(X)]=\mathrm{E}\left[\frac{N}{M n} Y g(Y-1)\right] .
$$

Proof. By the definition of expectation, we have

$$
\begin{aligned}
\mathrm{E}[g(X)] & =\sum_{x=0}^{n-1} g(x) P(X=x) \\
& =\sum_{x=0}^{n-1} g(x) \frac{\left(\begin{array}{c}
M-1 \\
x
\end{array}\right)\left(\begin{array}{c}
N-M \\
n-1-x
\end{array}\right)}{\left(\begin{array}{c}
N-1 \\
n-1
\end{array}\right)} \\
& \left.=\sum_{y=1}^{n} g(y-1) \frac{\left(\begin{array}{c}
M-1 \\
y-1
\end{array}\right)\left(\begin{array}{c}
N-M \\
n-y
\end{array}\right)}{\left(\begin{array}{c}
N-1 \\
n-1
\end{array}\right)} \quad \text { let } y=x+1\right) .
\end{aligned}
$$


According to the identities of combinatorial numbers

$$
\left(\begin{array}{c}
M-1 \\
y-1
\end{array}\right)=\frac{y}{M}\left(\begin{array}{c}
M \\
y
\end{array}\right) \text { and }\left(\begin{array}{c}
N-1 \\
n-1
\end{array}\right)=\frac{n}{N}\left(\begin{array}{c}
N \\
n
\end{array}\right),
$$

we obtain

$$
\begin{aligned}
\mathrm{E}[g(X)] & =\sum_{y=1}^{n} g(y-1) y \frac{N}{M n} \frac{\left(\begin{array}{c}
M \\
y
\end{array}\right)\left(\begin{array}{c}
N-M \\
n-y
\end{array}\right)}{\left(\begin{array}{c}
N \\
n
\end{array}\right)} \\
& \left.=\sum_{y=0}^{n} g(y-1) y \frac{N}{M n} \frac{\left(\begin{array}{c}
M \\
y
\end{array}\right)\left(\begin{array}{c}
N-M \\
n-y
\end{array}\right)}{\left(\begin{array}{c}
N \\
n
\end{array}\right)} \text { (the added term is } 0\right) \\
& =\sum_{y=0}^{n} g(y-1) y \frac{N}{M n} P(Y=y) \\
& =\mathrm{E}\left[\frac{N}{M n} Y g(Y-1)\right] .
\end{aligned}
$$

The proof of the theorem is complete.

\section{Using the Expectation Identity of the Hypergeometric Distribu- tion to Calculate Its High-Order Origin Moments}

In this section, we will use the expectation identity of the hypergeometric distribution to calculate its high-order origin moments. First, we will use the expectation identity of the hypergeometric distribution to calculate its first four origin moments. Second, we will use the expectation identity of the hypergeometric distribution to calculate its general $k$ th $(k=1,2, \ldots)$ origin moment. Third, we will use the general $k$ th origin moment to validate the first four origin moments of the hypergeometric distribution. Finally, the coefficients of the first 10 origin moments of the hypergeometric distribution are summarized in a table.

3.1. The analytical calculations of the first four origin moments of the hypergeometric distribution by using the expectation identity

In this subsection, we will analytically calculate the first four origin moments of the hypergeometric distribution $Y \sim H G(N, M, n)$ by the expectation identity 2 . 
First, let us calculate $\mathrm{E} Y$. Let $g(x)=(1+x)^{0}=1$. By the expectation identity (2), we have

$$
\mathrm{E} 1=\mathrm{E}\left(\frac{N}{M n} Y 1\right)
$$

Hence,

$$
\mathrm{E} Y=\frac{M n}{N}
$$

Therefore,

$$
\mathrm{E} X=\frac{(M-1)(n-1)}{N-1},
$$

which can be obtained by replacing $N, M$, and $n$ by $N-1, M-1$, and $n-1$ in the formula of $\mathrm{EY}(3)$.

Second, let us calculate $\mathrm{E} Y^{2}$. Let $g(x)=(1+x)^{1}$. By the expectation identity (2), we have

$$
\mathrm{E}(1+X)=\mathrm{E}\left(\frac{N}{M n} Y Y\right)
$$

Hence, by (4), we obtain

$$
\begin{aligned}
\mathrm{E} Y^{2} & =\frac{M n}{N} \mathrm{E}(1+X) \\
& =\frac{M n}{N}(1+\mathrm{E} X) \\
& =\frac{M n}{N}+\frac{M n}{N} \frac{(M-1)(n-1)}{N-1} \\
& =\frac{M n}{N}+\frac{M(M-1) n(n-1)}{N(N-1)} .
\end{aligned}
$$

Therefore,

$$
\mathrm{E} X^{2}=\frac{(M-1)(n-1)}{N-1}+\frac{(M-1)(M-2)(n-1)(n-2)}{(N-1)(N-2)},
$$

which can be obtained by replacing $N, M$, and $n$ by $N-1, M-1$, and $n-1$ in the formula of $\mathrm{EY}^{2}(5)$.

Third, let us calculate $\mathrm{E} Y^{3}$. Let $g(x)=(1+x)^{2}$. By the expectation identity (2), we have

$$
\mathrm{E}(1+X)^{2}=\mathrm{E}\left(\frac{N}{M n} Y Y^{2}\right)
$$


Hence, by (4) and (6), we obtain

$$
\begin{aligned}
\mathrm{E} Y^{3} & =\frac{M n}{N} \mathrm{E}(1+X)^{2} \\
& =\frac{M n}{N} \mathrm{E}\left(1+2 X+X^{2}\right) \\
& =\frac{M n}{N}\left(1+2 \mathrm{E} X+\mathrm{E} X^{2}\right) \\
& =\frac{M n}{N}\left[1+2 \frac{(M-1)(n-1)}{N-1}+\frac{(M-1)(n-1)}{N-1}+\frac{(M-1)(M-2)(n-1)(n-2)}{(N-1)(N-2)}\right] \\
& =\frac{M n}{N}+3 \frac{M(M-1) n(n-1)}{N(N-1)}+\frac{M(M-1)(M-2) n(n-1)(n-2)}{N(N-1)(N-2)} .
\end{aligned}
$$

Therefore,

$$
\begin{aligned}
\mathrm{E} X^{3}= & \frac{(M-1)(n-1)}{N-1}+3 \frac{(M-1)(M-2)(n-1)(n-2)}{(N-1)(N-2)} \\
& +\frac{(M-1)(M-2)(M-3)(n-1)(n-2)(n-3)}{(N-1)(N-2)(N-3)},
\end{aligned}
$$

which can be obtained by replacing $N, M$, and $n$ by $N-1, M-1$, and $n-1$ in the formula of $\mathrm{EY}^{3}(7)$.

Fourth, let us calculate $\mathrm{E} Y^{4}$. Let $g(x)=(1+x)^{3}$. By the expectation identity (2), we have

$$
\mathrm{E}(1+X)^{3}=\mathrm{E}\left(\frac{N}{M n} Y Y^{3}\right) .
$$

Hence, by (4), (6), and (8), we obtain

$$
\begin{aligned}
\mathrm{E} Y^{4}= & \frac{M n}{N} \mathrm{E}(1+X)^{3} \\
= & \frac{M n}{N} \mathrm{E}\left(1+3 X+3 X^{2}+X^{3}\right) \\
= & \frac{M n}{N}\left(1+3 \mathrm{E} X+3 \mathrm{E} X^{2}+\mathrm{E}^{3}\right) \\
= & \frac{M n}{N}\left[\begin{array}{c}
1+3 \frac{(M-1)(n-1)}{N-1}+3 \frac{(M-1)(n-1)}{N-1}+3 \frac{(M-1)(M-2)(n-1)(n-2)}{(N-1)(N-2)} \\
+\frac{(M-1)(n-1)}{N-1}+3 \frac{(M-1)(M-2)(n-1)(n-2)}{(N-1)(N-2)}+\frac{(M-1)(M-2)(M-3)(n-1)(n-2)(n-3)}{(N-1)(N-2)(N-3)}
\end{array}\right] \\
= & \frac{M n}{N}+7 \frac{M(M-1) n(n-1)}{N(N-1)}+6 \frac{M(M-1)(M-2) n(n-1)(n-2)}{N(N-1)(N-2)} \\
& +\frac{M(M-1)(M-2)(M-3) n(n-1)(n-2)(n-3)}{N(N-1)(N-2)(N-3)} .
\end{aligned}
$$


Therefore,

$$
\begin{aligned}
\mathrm{E} X^{4}= & \frac{(M-1)(n-1)}{N-1}+7 \frac{(M-1)(M-2)(n-1)(n-2)}{(N-1)(N-2)} \\
& +6 \frac{(M-1)(M-2)(M-3)(n-1)(n-2)(n-3)}{(N-1)(N-2)(N-3)} \\
& +\frac{(M-1)(M-2)(M-3)(M-4)(n-1)(n-2)(n-3)(n-4)}{(N-1)(N-2)(N-3)(N-4)},
\end{aligned}
$$

which can be obtained by replacing $N, M$, and $n$ by $N-1, M-1$, and $n-1$ in the formula of $\mathrm{E}^{4}$ (9).

It is easy to see that our analytical formulas of the first four origin moments of the hypergeometric distribution by using the expectation identity (2) are the same as those obtained in $\mathrm{Li}$ and Tian (2010), in which they use the definition of expectation for the calculations.

\subsection{The analytical calculations of the $k$ th origin moment of the hypergeometric}

distribution by using the expectation identity

In this subsection, we will analytically calculate the $k$ th origin moment of the hypergeometric distribution $Y \sim H G(N, M, n)$ by the expectation identity (2).

Define

$$
H_{k}=\frac{\left[\prod_{i=0}^{k}(M-i)\right]\left[\prod_{i=0}^{k}(n-i)\right]}{\prod_{i=0}^{k}(N-i)}, k=0,1,2,3, \ldots
$$

In particular,

$$
\begin{aligned}
& H_{0}=\frac{M n}{N}, \\
& H_{1}=\frac{M(M-1) n(n-1)}{N(N-1)}, \\
& H_{2}=\frac{M(M-1)(M-2) n(n-1)(n-2)}{N(N-1)(N-2)}, \\
& H_{3}=\frac{M(M-1)(M-2)(M-3) n(n-1)(n-2)(n-3)}{N(N-1)(N-2)(N-3)} .
\end{aligned}
$$


From the calculation results in the previous section, we find that the first four origin moments of the hypergeometric distribution $Y \sim H G(N, M, n)$ can be written as a linear combination of $H_{0}, H_{1}, H_{2}$, and $H_{3}$. This fact inspires us to claim that the general $k$ th origin moment of $Y \sim H G(N, M, n)$, $\mathrm{E} Y^{k}(k=0,1,2, \ldots)$, can also be written as a linear combination of $H_{0}, H_{1}$, $\ldots, H_{k-1}$ given in (11).

To prove Theorem 2, we need the following lemma, which gives a relationship between the expressions of $\mathrm{E} Y^{k}$ and $(M n / N) \mathrm{E} X^{k}$.

Lemma 1. Let $X \sim H G(N-1, M-1, n-1)$ and $Y \sim H G(N, M, n)$. If

$$
\mathrm{E} Y^{k}=\sum_{j=1}^{k} a_{j} H_{j-1}
$$

is a linear combination of $H_{0}, H_{1}, \ldots, H_{k-1}$ given in 11, then

$$
\frac{M n}{N} \mathrm{E} X^{k}=\sum_{j=1}^{k} a_{j} H_{j}
$$

is a linear combination of $H_{1}, H_{2}, \ldots, H_{k}$.

Proof. If

$$
\begin{aligned}
\mathrm{E} Y^{k} & =\sum_{j=1}^{k} a_{j} H_{j-1} \\
& =\sum_{j=1}^{k} a_{j} \frac{\left[\prod_{i=0}^{j-1}(M-i)\right]\left[\prod_{i=0}^{j-1}(n-i)\right]}{\prod_{i=0}^{j-1}(N-i)}
\end{aligned}
$$

is a linear combination of $H_{0}, H_{1}, \ldots, H_{k-1}$, then

$$
\mathrm{E} X^{k}=\sum_{j=1}^{k} a_{j} \frac{\left[\prod_{i=0}^{j-1}(M-1-i)\right]\left[\prod_{i=0}^{j-1}(n-1-i)\right]}{\prod_{i=0}^{j-1}(N-1-i)},
$$


which can be obtained by replacing $N, M$, and $n$ by $N-1, M-1$, and $n-1$ in the formula of $\mathrm{E}^{k}$ 12. Hence,

$$
\begin{aligned}
\frac{M n}{N} \mathrm{E} X^{k} & =\sum_{j=1}^{k} a_{j} \frac{\left[M \prod_{i=0}^{j-1}(M-1-i)\right]\left[n \prod_{i=0}^{j-1}(n-1-i)\right]}{N \prod_{i=0}^{j-1}(N-1-i)} \\
& =\sum_{j=1}^{k} a_{j} \frac{M(M-1) \cdots(M-j) n(n-1) \cdots(n-j)}{N(N-1) \cdots(N-j)} \\
& =\sum_{j=1}^{k} a_{j} \frac{\left[\prod_{i=0}^{j}(M-i)\right]\left[\prod_{i=0}^{j}(n-i)\right]}{\prod_{i=0}^{j}(N-i)} \\
& =\sum_{j=1}^{k} a_{j} H_{j},
\end{aligned}
$$

is a linear combination of $H_{1}, H_{2}, \ldots, H_{k}$.

The proof of the lemma is complete.

By Lemma 1 and the mathematical induction method, we have the following theorem, in which $\mathrm{E} Y^{k}$ is expressed as a linear combination of $H_{0}, H_{1}, \ldots, H_{k-1}$ given in 11.

Theorem 2. Let $Y \sim H G(N, M, n)$. Then

$$
\mathrm{E} Y^{k}=\sum_{j=1}^{k} A(k, j) H_{j-1},
$$

is a linear combination of $H_{0}, H_{1}, \ldots, H_{k-1}$ given in (11), where the coefficients $A(k, j)$ are given by the following recurrence formula:

$$
A(k, j)= \begin{cases}1, & k=j \text { or } j=1, \\
\sum_{i=j-1}^{k-1}\left(\begin{array}{c}
k-1 \\
i
\end{array}\right) A(i, j-1), & k>j>1 .\end{cases}
$$

Proof. We will use the mathematical induction method to prove this theorem. 
1. When $k=1$,

$$
\mathrm{E} Y=\frac{M n}{N}=H_{0}=A(1,1) H_{0}=\sum_{j=1}^{1} A(1, j) H_{j-1},
$$

and thus 13 is correct.

2. Suppose that (13) is true when $1 \leq k \leq l$. According to Lemma 1, we have

$$
\frac{M n}{N} \mathrm{E} X^{k}=\sum_{j=1}^{k} A(k, j) H_{j},
$$

for $1 \leq k \leq l$.

3. When $k=l+1$, let $g(x)=(1+x)^{l}$. By expectation identity (2), we have

$$
\mathrm{E}(1+X)^{l}=\mathrm{E}\left(\frac{N}{M n} Y Y^{l}\right) .
$$

Hence, by [15], we obtain

$$
\begin{aligned}
\mathrm{E} Y^{l+1}= & \frac{M n}{N} \mathrm{E}(1+X)^{l} \\
= & \frac{M n}{N} \mathrm{E}\left[\left(\begin{array}{l}
l \\
0
\end{array}\right) X^{0}+\left(\begin{array}{l}
l \\
1
\end{array}\right) X^{1}+\left(\begin{array}{l}
l \\
2
\end{array}\right) X^{2}+\left(\begin{array}{l}
l \\
3
\end{array}\right) X^{3}+\cdots+\left(\begin{array}{c}
l \\
l-1
\end{array}\right) X^{l-1}+\left(\begin{array}{l}
l \\
l
\end{array}\right) X^{l}\right] \\
= & \frac{M n}{N}\left[\left(\begin{array}{l}
l \\
0
\end{array}\right)+\left(\begin{array}{l}
l \\
1
\end{array}\right) \mathrm{E} X^{1}+\left(\begin{array}{l}
l \\
2
\end{array}\right) \mathrm{E} X^{2}+\left(\begin{array}{l}
l \\
3
\end{array}\right) \mathrm{E} X^{3}+\cdots+\left(\begin{array}{c}
l \\
l-1
\end{array}\right) \mathrm{E} X^{l-1}+\left(\begin{array}{l}
l \\
l
\end{array}\right) \mathrm{E} X^{l}\right] \\
= & \frac{M n}{N}+\left(\begin{array}{l}
l \\
1
\end{array}\right) \frac{M n}{N} \mathrm{E} X^{1}+\left(\begin{array}{l}
l \\
2
\end{array}\right) \frac{M n}{N} \mathrm{E} X^{2}+\left(\begin{array}{l}
l \\
3
\end{array}\right) \frac{M n}{N} \mathrm{E} X^{3}+\cdots \\
& +\left(\begin{array}{c}
l \\
l-1
\end{array}\right) \frac{M n}{N} \mathrm{E} X^{l-1}+\left(\begin{array}{l}
l \\
l
\end{array}\right) \frac{M n}{N} \mathrm{E} X^{l} \\
= & H_{0}+\left(\begin{array}{l}
l \\
1
\end{array}\right)\left[A(1,1) H_{1}\right]+\left(\begin{array}{l}
l \\
2
\end{array}\right)\left[A(2,1) H_{1}+A(2,2) H_{2}\right] \\
& +\left(\begin{array}{l}
l \\
3
\end{array}\right)\left[A(3,1) H_{1}+A(3,2) H_{2}+A(3,3) H_{3}\right]+\cdots \\
& +\left(\begin{array}{c}
l \\
l-1
\end{array}\right)\left[\begin{array}{l}
l-1 \\
j=1
\end{array}\right] \\
= & H_{0}+\sum_{i=1}^{l}\left(\begin{array}{l}
l \\
i
\end{array}\right) A(i, 1) H_{1}+\sum_{i=2}^{l}\left(\begin{array}{l}
l \\
i
\end{array}\right) A(i, 2) H_{2}+\cdots+\sum_{i=l}^{l}\left(\begin{array}{l}
l \\
i
\end{array}\right) A(i, l) H_{l} \\
= & A(l+1,1) H_{0}+A(l+1,2) H_{1}+A(l+1,3) H_{2}+\cdots+A(l+1, l+1) H_{l} \\
& l+1 \\
l & \sum_{j=1}^{l} A(l+1, j) H_{j-1} .
\end{aligned}
$$


Therefore, when $k=l+1,(13)$ also holds true.

In conclusion, by the mathematical induction method, 13 is established and the proof of the theorem is completed.

\subsection{Verifications of the first four origin moments by the kth origin moment}

In this subsection, we will verify the first four origin moments of the hypergeometric distribution $Y \sim H G(N, M, n)$ by the $k$ th origin moment 13$)$. The verifications ensure that the general formula for the $k$ th origin moment 13 is correct.

(1) When $k=1$,

$$
\begin{aligned}
\mathrm{E} Y & =\sum_{j=1}^{1} A(1, j) H_{j-1} \\
& =A(1,1) H_{0} \\
& =H_{0},
\end{aligned}
$$

which is the same as in 3 .

(2) When $k=2$,

$$
\begin{aligned}
\mathrm{E} Y^{2} & =\sum_{j=1}^{2} A(2, j) H_{j-1} \\
& =A(2,1) H_{0}+A(2,2) H_{1} \\
& =H_{0}+H_{1},
\end{aligned}
$$

which is the same as in (5).

(3) When $k=3$,

$$
\begin{aligned}
\mathrm{E} Y^{3} & =\sum_{j=1}^{3} A(3, j) H_{j-1} \\
& =A(3,1) H_{0}+A(3,2) H_{1}+A(3,3) H_{2} .
\end{aligned}
$$

According to 14,

$$
A(3,1)=A(3,3)=1
$$


and

$$
A(3,2)=\sum_{i=1}^{2}\left(\begin{array}{l}
2 \\
i
\end{array}\right) A(i, 1)=\left(\begin{array}{l}
2 \\
1
\end{array}\right) A(1,1)+\left(\begin{array}{l}
2 \\
2
\end{array}\right) A(2,1)=3 .
$$

Therefore,

$$
\mathrm{E} Y^{3}=H_{0}+3 H_{1}+H_{2},
$$

which is the same as in (7).

(4) When $k=4$,

$$
\begin{aligned}
\mathrm{E} Y^{4} & =\sum_{j=1}^{4} A(4, j) H_{j-1} \\
& =A(4,1) H_{0}+A(4,2) H_{1}+A(4,3) H_{2}+A(4,4) H_{3} .
\end{aligned}
$$

According to 14,

$$
\begin{gathered}
A(4,1)=A(4,4)=1, \\
A(4,2)=\sum_{i=1}^{3}\left(\begin{array}{l}
3 \\
i
\end{array}\right) A(i, 1)=\left(\begin{array}{l}
3 \\
1
\end{array}\right) A(1,1)+\left(\begin{array}{l}
3 \\
2
\end{array}\right) A(2,1)+\left(\begin{array}{l}
3 \\
3
\end{array}\right) A(3,1)=7,
\end{gathered}
$$

and

$$
A(4,3)=\sum_{i=2}^{3}\left(\begin{array}{l}
3 \\
i
\end{array}\right) A(i, 2)=\left(\begin{array}{l}
3 \\
2
\end{array}\right) A(2,2)+\left(\begin{array}{l}
3 \\
3
\end{array}\right) A(3,2)=6
$$

Therefore,

$$
\mathrm{E} Y^{4}=H_{0}+7 H_{1}+6 H_{2}+H_{3},
$$

which is the same as in (9).

3.4. The coefficients table of the kth origin moment of the hypergeometric distribution

The coefficients of the first 10 origin moments of the hypergeometric distribution $Y \sim H G(N, M, n)$ are shown in Table 1 .

Comparing Table 1 with the table of Stirling numbers of the second kind and exponential numbers (P310) in Comtet (1974) and Table 1 in Li and Tian (2010), we find that

$$
A(k, j)=S(k, j)
$$


Table 1: The coefficients of the first 10 origin moments of the hypergeometric distribution $Y \sim H G(N, M, n)$.

\begin{tabular}{|r|c|c|c|c|c|c|c|c|c|c|}
\hline & $j=1$ & 2 & 3 & 4 & 5 & 6 & 7 & 8 & 9 & 10 \\
\hline$k=1$ & 1 & & & & & & & & & \\
\hline 2 & 1 & 1 & & & & & & & & \\
\hline 3 & 1 & 3 & 1 & & & & & & & \\
\hline 4 & 1 & 7 & 6 & 1 & & & & & & \\
\hline 5 & 1 & 15 & 25 & 10 & 1 & & & & & \\
\hline 6 & 1 & 31 & 90 & 65 & 15 & 1 & & & & \\
\hline 7 & 1 & 63 & 301 & 350 & 140 & 21 & 1 & & & \\
\hline 8 & 1 & 127 & 966 & 1701 & 1050 & 266 & 28 & 1 & & \\
\hline 9 & 1 & 255 & 3025 & 7770 & 6951 & 2646 & 462 & 36 & 1 & \\
\hline 10 & 1 & 511 & 9330 & 34105 & 42525 & 22827 & 5880 & 750 & 45 & 1 \\
\hline
\end{tabular}

are Stirling numbers of the second kind. Moreover, the $A(k, j)$ given by (14 satisfy the "vertical" recurrence relations given in Theorem B [3c] (P209) in Comtet (1974), and this fact further reassures that $A(k, j)$ are Stirling numbers of the second kind.

Therefore, Theorem 2 can be restated by the following theorem in the symbol of $S(k, j)$, which are Stirling numbers of the second kind.

Theorem 3. Let $Y \sim H G(N, M, n)$. Then

$$
\mathrm{E} Y^{k}=\sum_{j=1}^{k} S(k, j) H_{j-1},
$$

is a linear combination of $H_{0}, H_{1}, \ldots, H_{k-1}$ given in (11), where the coefficients $S(k, j)$ are Stirling numbers of the second kind. 


\section{Numerical, Theoretical Results}

In this section, we will provide an assessment of the computation complexity (see Chapter 5 of Progri (2011)) which is given in number of multiplications of the Definition Method (DM) (1) and the Analytical Method (AM) (16). Moreover, we will investigate the memory size of the DM and the AM. Furthermore, we will carry out some numerical experiments to compare the computations of $\mathrm{E} Y^{k}$ and the computing times by the DM and the AM. Finally, we will compare the absolute errors and the relative errors between the two methods. See the Numerical, Theoretical Results section of Progri $(2021 \mathrm{a} \mid \mathrm{b}$ c $)$ for details. The numerical experiments are carried out on a Lenovo computer 11th Gen Intel(R) Core(TM) i5-11300H @ 3.10GHz with 16.0 GB RAM using R version 4.1.1.

Now let us provide an assessment of the computation complexity which is given in number of multiplications of the DM and the AM. For the DM (1),

$$
\left(\begin{array}{c}
M \\
y
\end{array}\right)=\frac{M !}{y !(M-y) !}=\frac{M(M-1) \cdots(M-y+1)}{y !}
$$

which can be computed in $\mathcal{O}(2 y-1)$. Similarly, $\left(\begin{array}{c}N-M \\ n-y\end{array}\right)$ can be computed in $\mathcal{O}(2(n-y)-1)$, and $\left(\begin{array}{l}N \\ n\end{array}\right)$ can be computed in $\mathcal{O}(2 n-1)$. Moreover, $y^{k}$ can be computed in $\mathcal{O}(k-1)$. Hence,

$$
y^{k} \frac{\left(\begin{array}{c}
M \\
y
\end{array}\right)\left(\begin{array}{c}
N-M \\
n-y
\end{array}\right)}{\left(\begin{array}{c}
N \\
n
\end{array}\right)}
$$

can be computed in

$$
\begin{aligned}
& \mathcal{O}(k-1)+\mathcal{O}(2 y-1)+\mathcal{O}(2(n-y)-1)+\mathcal{O}(2 n-1)+\mathcal{O}(3) \\
& =\mathcal{O}(k+4 n-1)=\mathcal{O}(k+4 n)
\end{aligned}
$$

Therefore, $\mathrm{E} Y^{k}$ by the DM can be computed in

$$
(n+1) \mathcal{O}(k+4 n)=\mathcal{O}((n+1)(k+4 n))=\mathcal{O}(n(k+4 n)) .
$$

For the AM (16), $S(k, j)$ can be precomputed and stored in a lower-triangular matrix $S$ of size $k \times k$, which is independent of $N, M$, and $n$, and thus the computation cost of $S(k, j)$ can be regarded as 0 . Note that $H_{k}$ 11 can 
be computed in $\mathcal{O}(3 k+2)$, and thus $H_{j-1}$ can be computed in $\mathcal{O}(3 j-1)$. Therefore, $\mathrm{E} Y^{k}$ by the AM can be computed in

$$
\sum_{j=1}^{k} \mathcal{O}(3 j)=\mathcal{O}\left(\sum_{j=1}^{k} 3 j\right)=\mathcal{O}\left(3 \sum_{j=1}^{k} j\right)=\mathcal{O}\left(\frac{3}{2} k(k+1)\right)=\mathcal{O}\left(\frac{3}{2} k^{2}\right) .
$$

Now let us investigate the memory size of the DM and the AM. For the DM, we only need to store a scalar, and thus the memory size of the DM is 1 double floating-point number. For the AM, we need to store two vectors of length $k$, and a precomputed lower-triangular matrix $S$ of size $k \times k$. Therefore, the memory size of the AM is

$$
k+k+\frac{1}{2} k(k+1)=\frac{1}{2} k(k+5)
$$

double floating-point numbers. In most cases, $k$ would be small, and thus the memory size of the AM would be small.

In summary, compared to the DM, the AM would be computationally more efficient, but suffers from a heavier burden of memory size.

The numerical values of $\mathrm{E}^{k}(k=1, \ldots, 10)$ and the computing times (in seconds, 10,000 replications) by the two methods (DM and AM) for $N=1000$, $M=500$, and $n=10$ are summarized in Table 2 From the table, we see that $\mathrm{E} Y^{k}$ increases as $k$ increases for other parameters fixed. Moreover, the two methods produce the same answers accurate to two decimal points. To obtain reliable computing times, we have done 10,000 replications, that is, we have computed each moment for 10,000 times, and the total computing times in seconds are reported in the table. Therefore, for each moment in a single replication, the computing time is $1 / 10,000$ of the computing time reported in the table. For example, the computing time for $\mathrm{EY}^{1}$ for once by the DM is

$$
\frac{1.71}{10,000} \text { seconds }=\frac{1.71}{10} \text { milliseconds }=0.171 \text { milliseconds } .
$$

Hence, the computing for each moment by either method is very fast. Finally, the computation of $\mathrm{E} Y^{k}$ by the AM is consistently faster than that by the DM.

Now let us consider the absolute errors and the relative errors between the $\mathrm{DM}$ and the AM. The absolute error between the two methods to compute $\mathrm{E} Y^{k}$ 
Table 2: The numerical values of $\mathrm{E}^{k}(k=1, \ldots, 10)$ and the computing times (in seconds, 10,000 replications) by the two methods (DM and AM) for $N=$ $1000, M=500$, and $n=10$.

\begin{tabular}{|r|r|r|l|l|}
\hline & \multicolumn{2}{|c|}{$\mathrm{EY}^{k}$} & \multicolumn{2}{c|}{ Computing Times } \\
\hline$k$ & $\mathrm{DM}$ & $\mathrm{AM}$ & $\mathrm{DM}$ & $\mathrm{AM}$ \\
\hline 1 & 5.00 & 5.00 & 1.71 & 1.50 \\
\hline 2 & 27.48 & 27.48 & 1.66 & 1.53 \\
\hline 3 & 162.16 & 162.16 & 1.63 & 1.50 \\
\hline 4 & 1013.83 & 1013.83 & 1.61 & 1.47 \\
\hline 5 & 6652.05 & 6652.05 & 1.60 & 1.47 \\
\hline 6 & 45490.02 & 45490.02 & 1.63 & 1.54 \\
\hline 7 & 322491.55 & 322491.55 & 1.64 & 1.49 \\
\hline 8 & 2359910.34 & 2359910.34 & 1.59 & 1.48 \\
\hline 9 & 17762842.81 & 17762842.81 & 1.61 & 1.49 \\
\hline 10 & 137113809.34 & 137113809.34 & 1.69 & 1.51 \\
\hline
\end{tabular}


is

$$
A E\left(\mathrm{E} Y^{k}\right)=\left|\mathrm{E} Y_{D M}^{k}-\mathrm{E} Y_{A M}^{k}\right|
$$

where $\mathrm{E} Y_{D M}^{k}$ and $\mathrm{E} Y_{A M}^{k}$ are the $\mathrm{E} Y^{k}$ values computed by the DM and the AM, respectively. The relative error between the two methods to compute $\mathrm{E} Y^{k}$ is

$$
R E\left(\mathrm{E} Y^{k}\right)=\frac{\left|\mathrm{E} Y_{D M}^{k}-\mathrm{E} Y_{A M}^{k}\right|}{\mathrm{E} Y_{A M}^{k}}=\frac{A E\left(\mathrm{E} Y^{k}\right)}{\mathrm{E} Y_{A M}^{k}} .
$$

The absolute errors and the relative errors between the two methods to compute $\mathrm{E} Y^{k}$ for $k=1, \ldots, 20$ with $N=1000, M=500$, and $n=10$ are reported in Table 3 . From the table, we see that the absolute errors between the two methods to compute $\mathrm{EY}^{k}$ are quite small except for $k=18$ and 20, and the absolute errors are 1 and 32 , respectively. However, since $\mathrm{EY}^{k}$ for $k=18$ and 20 are extremely large,

$$
\begin{aligned}
& \mathrm{E} Y_{A M}^{18}=3352936804349472.00 \\
& \mathrm{E}_{A M}^{20}=268954341041673952.00
\end{aligned}
$$

the relative errors between the two methods to compute $\mathrm{E} Y^{k}$ are still acceptably small,

$$
R E\left(\mathrm{E} Y^{18}\right)=2.98 \mathrm{e}-16 \text { and } R E\left(\mathrm{E} Y^{20}\right)=1.19 \mathrm{e}-16
$$

Now let us carry out some sensitivity analysis to compute $\mathrm{E}^{k}$ by allowing one of the parameters $(k, N, M$, and $n)$ to change, holding other parameters fixed. The parameter values are fixed as

$$
k=10, N=1000, M=500, \text { and } n=10 .
$$

Other parameter values can also be specified.

The sensitivity analysis of $\mathrm{EY}^{k}$ by the DM and the AM against the parameters $k, N, M$, and $n$ are plotted in Figure 1. From the figure, we see that $\mathrm{E} Y^{k}$ are increasing functions of $k, M$, and $n$, and it is a decreasing function of $N$. The two curves in the plots are indistinguishable, which means that the two methods produce almost the same answers to $\mathrm{E} Y^{k}$. It is worth noting that $\mathrm{E} Y^{k}$ for $n \geq 385$ by the $\mathrm{DM}$ are $\infty$ (see the lower right plot), while the AM 
Table 3: The absolute errors and the relative errors between the two methods to compute $\mathrm{E}^{k}$ for $k=1, \ldots, 20$ with $N=1000, M=500$, and $n=10$.

\begin{tabular}{|c|c|c|c|c|c|}
\hline$k$ & 1 & 2 & 3 & 4 & 5 \\
\hline AE & $8.88 \mathrm{e}-16$ & $3.55 \mathrm{e}-15$ & $2.84 \mathrm{e}-14$ & $1.14 \mathrm{e}-13$ & $9.09 \mathrm{e}-13$ \\
\hline RE & $1.78 \mathrm{e}-16$ & $1.29 \mathrm{e}-16$ & $1.75 \mathrm{e}-16$ & $1.12 \mathrm{e}-16$ & $1.37 \mathrm{e}-16$ \\
\hline$k$ & 6 & 7 & 8 & 9 & 10 \\
\hline AE & $7.28 \mathrm{e}-12$ & $0.00 \mathrm{e}+00$ & $4.66 \mathrm{e}-10$ & $3.73 \mathrm{e}-09$ & $2.98 \mathrm{e}-08$ \\
\hline RE & $1.60 \mathrm{e}-16$ & $0.00 \mathrm{e}+00$ & $1.97 \mathrm{e}-16$ & $2.10 \mathrm{e}-16$ & $2.17 \mathrm{e}-16$ \\
\hline$k$ & 11 & 12 & 13 & 14 & 15 \\
\hline AE & $0.00 \mathrm{e}+00$ & $0.00 \mathrm{e}+00$ & $0.00 \mathrm{e}+00$ & $0.00 \mathrm{e}+00$ & $9.77 \mathrm{e}-04$ \\
\hline $\mathrm{RE}$ & $0.00 \mathrm{e}+00$ & $0.00 \mathrm{e}+00$ & $0.00 \mathrm{e}+00$ & $0.00 \mathrm{e}+00$ & $1.92 \mathrm{e}-16$ \\
\hline$k$ & 16 & 17 & 18 & 19 & 20 \\
\hline $\mathrm{AE}$ & $0.00 \mathrm{e}+00$ & $0.00 \mathrm{e}+00$ & $1.00 \mathrm{e}+00$ & $0.00 \mathrm{e}+00$ & $3.20 \mathrm{e}+01$ \\
\hline $\mathrm{RE}$ & $0.00 \mathrm{e}+00$ & $0.00 \mathrm{e}+00$ & $2.98 \mathrm{e}-16$ & $0.00 \mathrm{e}+00$ & $1.19 \mathrm{e}-16$ \\
\hline
\end{tabular}


produces reasonable $\mathrm{E} Y^{k}$ values for $n \geq 385$. Therefore, the AM is more robust to extreme parameter values than the DM.

The sensitivity analysis of the absolute errors and the relative errors against the parameters $k, N, M$, and $n$ are plotted in Figure 2 . The upper left plot is a graphical version of Table 3 From the upper left plot, we see that the absolute errors of $\mathrm{EY}^{18}$ and $\mathrm{E} Y^{20}$ are large, however, their relative errors are acceptably small. Moreover, the absolute errors against $N$ and $M$ are acceptably small, and the relative errors against $N$ and $M$ are much smaller. Furthermore, the absolute errors against $n$ is very large, however, the relative errors against $n$ are acceptably small. All the plots indicate that the relative errors are acceptably small, and in fact, they are almost 0 .

\section{Conclusions and Discussions}

We have provided a noval method to calculate the high-order origin moments of the hypergeometric distribution $Y \sim H G(N, M, n)$, that is, the expectation identity method. First, the expectation identity of the hypergeometric distribution is discovered and summarized in Theorem 1. After that, we analytically calculate the first four origin moments of the hypergeometric distribution by using the expectation identity. Furthermore, we analytically calculate the general $k$ th $(k=1,2, \ldots)$ origin moment of the hypergeometric distribution by using the expectation identity, and the results are summarized in Theorem 2. Moreover, we use the general $k$ th origin moment to validate the first four origin moments of the hypergeometric distribution. The coefficients of the first 10 origin moments of the hypergeometric distribution are shown in Table 1. Comparing Table 1 with the tables in the literature, we find that $A(k, j)$ in Theorem 2 are Stirling numbers of the second kind. Finally, Theorem 2 is restated by Theorem 3 in the symbol of $S(k, j)$, which are Stirling numbers of the second kind.

We have provided an assessment of the computation complexity which is given in number of multiplications of the DM and the AM, which are respectively $\mathcal{O}(n(k+4 n))$ and $\mathcal{O}\left(3 k^{2} / 2\right)$. Moreover, we have investigated the memory size 

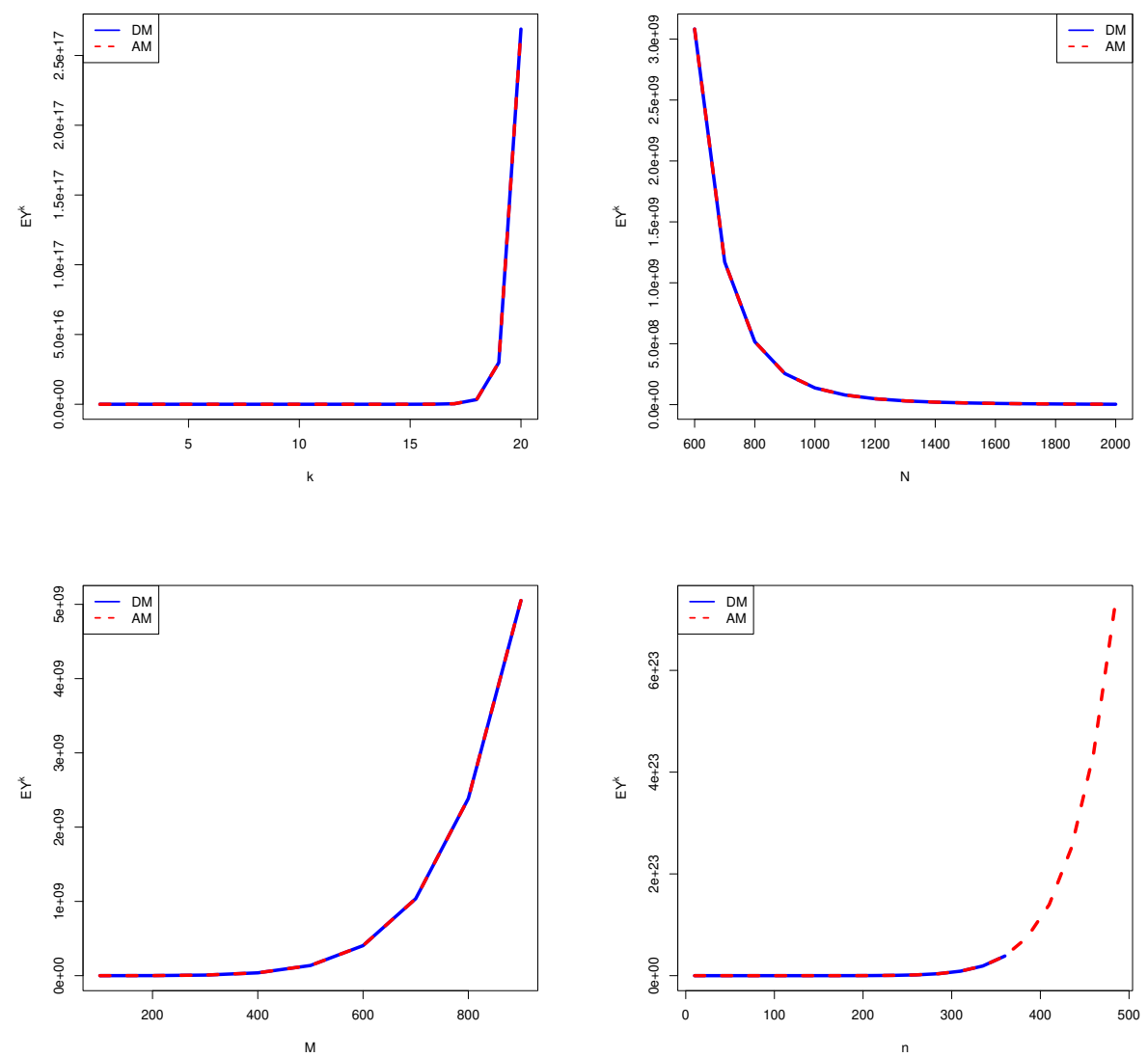

Figure 1: The sensitivity analysis of $\mathrm{E} Y^{k}$ by the DM and the AM against the parameters $k, N, M$, and $n$. 

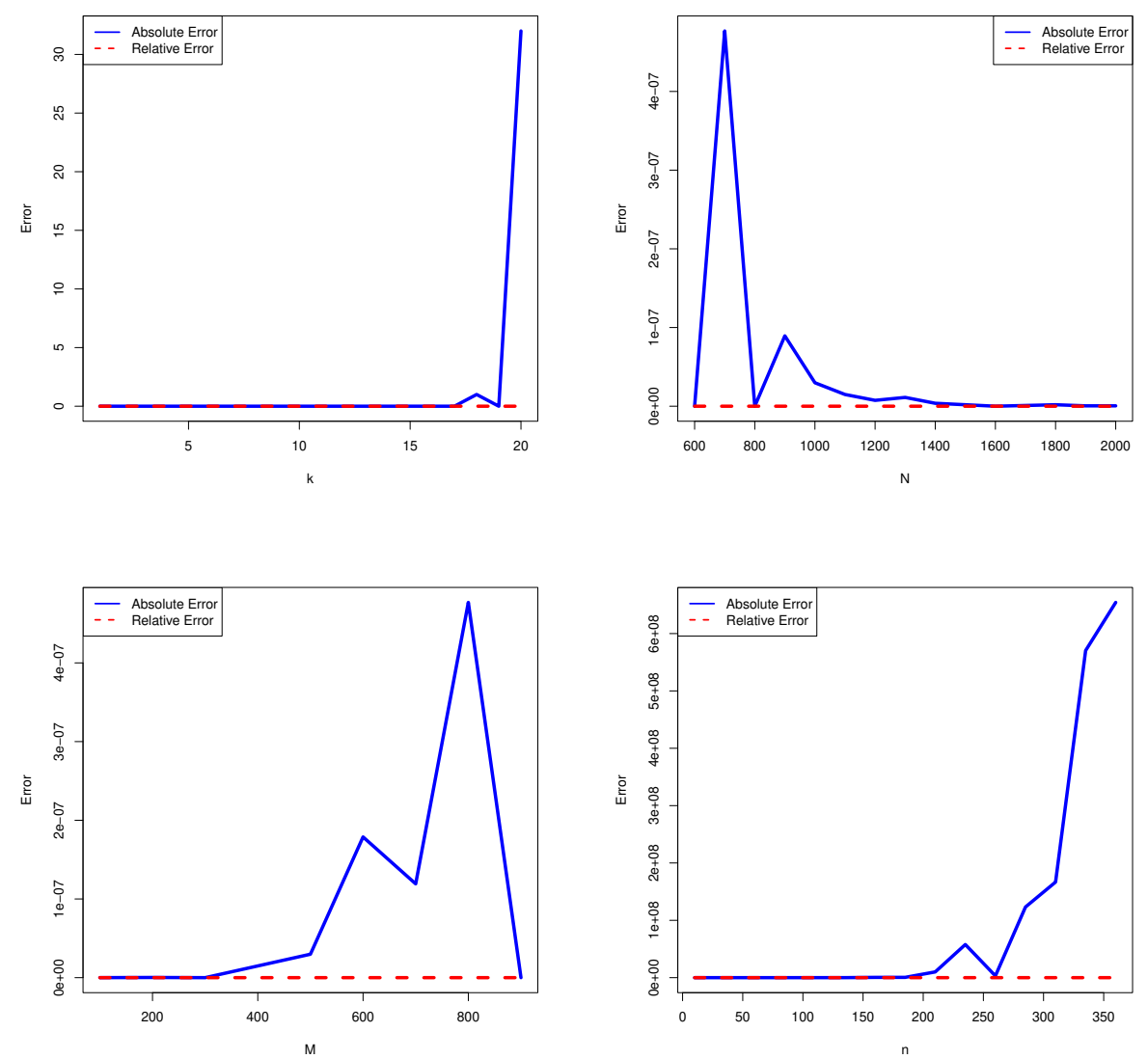

Figure 2: The sensitivity analysis of the absolute errors and the relative errors against the parameters $k, N, M$, and $n$. 
of the DM and the AM, which are respectively 1 and $k(k+5) / 2$ double floatingpoint numbers. Furthermore, we have carried out some numerical experiments to compare the computations of $\mathrm{E} Y^{k}$ and the computing times by the two methods. Finally, we have compared the absolute errors and the relative errors between the two methods. In summary, compared to the DM, the AM would be computationally more efficient, but suffers from a heavier burden of memory size. Moreover, the AM is more robust to extreme parameter values than the DM, as observed from the lower right plot in Figure 1.

\section{Acknowledgments}

The authors are extremely grateful to the editor, the associate editor, and the reviewer for their insightful comments that led to significant improvement of the article.

\section{Fundings}

The research was supported by the Ministry of Education (MOE) project of Humanities and Social Sciences on the west and the border area (20XJC910001), the National Social Science Fund of China (21XTJ001), the National Natural Science Foundation of China (72071019), and the Fundamental Research Funds for the Central Universities (2020CDJQY-Z001; 2021CDJQY-047).

\section{Supporting Information}

Additional information for this article is available.

The $\mathrm{R}$ codes used in the article can be downloaded from the link: https://pan.baidu.com/s/1SzEN1fHwnRvB8HzOdtpoxg, with password: 1234. Alternatively, the readers can send emails to the corresponding author. 


\section{References}

Brunk, H. D., Holstein, J. E., and Williams, F. (1968). A comparison of binomial approximations to hypergeometric distribution. The American Statistician, $22(1): 24-26$.

Casella, G. and Berger, R. L. (2002). Statistical Inference. Duxbury, Pacific Grove. 2nd edition.

Childs, A. and Balakrishnan, N. (2000). Some approximations to the multivariate hypergeometric distribution with applications to hypothesis testing. Computational Statistics \& Data Analysis, 35(2):137-154.

Chvatal, V. (1979). Tail of the hypergeometric distribution. Discrete Mathematics, 25(3):285-287.

Comtet, L. (1974). Advanced Combinatorics. D. Reidel Publishing Company, Boston. Revised and enlarged edition.

Danielian, E., Chitchyan, R., and Farbod, D. (2016). On a new regularly varying generalized hypergeometric distribution of the second type. Mathematical Reports, 18(2):217-232.

de Klerk, E., Laurent, M., and Sun, Z. (2015). An error analysis for polynomial optimization over the simplex based on the multivariate hypergeometric distribution. SIAM Journal on Optimization, 25(3):1498-1514.

Dinwoodie, I. H., Matusevich, L. F., and Mosteig, E. (2004). Transform methods for the hypergeometric distribution. Statistics and Computing, 14(4):287-297.

Eisinga, R. and Pelzer, B. (2011). Saddlepoint approximations to the mean and variance of the extended hypergeometric distribution. Statistica Neerlandica, $65(1): 22-31$.

Fog, A. (2008). Sampling methods for wallenius' and fisher's noncentral hypergeometric distributions. Communications in Statistics-Simulation and Computation, 37(2):241-257. 
Greene, E. and Wellner, J. A. (2017). Exponential bounds for the hypergeometric distribution. Bernoulli, 23(3):1911-1950.

Guenther, W. C. (1978). Some remarks on runs test and use of hypergeometric distribution. The American Statistician, 32(2):71-73.

Hafid, A., Hafid, A. S., and Samih, M. (2020). A novel methodology-based joint hypergeometric distribution to analyze the security of sharded blockchains. IEEE Access, 8:179389-179399.

Harkness, W. L. (1965). Properties of the extended hypergeometric distribution. Annals of Mathematical Statistics, 36(3):938-945.

Hida, E. and Akahira, M. (2003). An approximation to the generalized hypergeometric distribution. Statistical Papers, 44(4):483-497.

Hush, D. and Scovel, C. (2005). Concentration of the hypergeometric distribution. Statistics \& Probability Letters, 75(2):127-132.

Hwang, J. T. (1982). Improving on standard estimators in discrete exponential families with applications to poisson and negative binomial cases. The Annals of Statistics, 10:857-867.

Kachitvichyanukul, V. and Schmeiser, B. W. (1988). Algorithm 668 h2pec sampling from the hypergeometric distribution. ACM Transactions on Mathematical Software, 14(4):397-398.

Kemp, C. D. and Kemp, A. W. (1956). Generalized hypergeometric distributions. Journal of the Royal Statistical Society Series B-Statistical Methodology, 18(2):202-211.

Kochman, F., Murray, A., and West, D. B. (1989). An entropy inequality for the bi-multivariate hypergeometric distribution. Proceedings of the American Mathematical Society, 107(2):479-485. 
Krishnamoorthy, K. and Lv, S. (2020). Prediction intervals for hypergeometric distributions. Communications in Statistics-Theory and Methods, 49(6):15281536.

Kumar, C. S. (2002). Extended generalized hypergeometric probability distributions. Statistics $\&$ Probability Letters, 59(1):1-7.

Kumar, C. S. (2007). Some properties of bivariate generalized hypergeometric probability distributions. Journal of the Korean Statistical Society, 36(3):349355.

Lahiri, S. N., Chatterjee, A., and Maiti, T. (2007). Normal approximation to the hypergeometric distribution in nonstandard cases and a sub-gaussian berryesseen theorem. Journal of Statistical Planning and Inference, 137(11):35703590.

Lai, T. L. (1979). Sequential tests for hypergeometric distributions and finite populations. Annals of Statistics, 7(1):46-59.

Lebrun, R. (2013). Efficient time/space algorithm to compute rectangular probabilities of multinomial, multivariate hypergeometric and multivariate plya distributions. Statistics and Computing, 23(5):615-623.

Li, J. Q. and Tian, Q. J. (2010). Simple algorithm for the hypergeometric distribution moments about the origin of higher-order. Science Technology and Engineering, 10:7989-7992. In Chinese.

Lieberman, G. J. and Owen, D. B. (1962). Tables of the hypergeometric probability-distribution. Psychometrika, 27(3):321-322.

Lin, M. H. and Hsiung, C. A. (1994). Empirical bayes estimates of domain scores under binomial and hypergeometric distributions for test-scores. Psychometrika, 59(3):331-359.

Lin, M. H., Hsiung, C. A., and Hsiao, C. F. (1994). A program for monotonizing 2 empirical bayes estimators in binomial and hypergeometric data distributions. Psychometrika, 59(3):423-424. 
Ling, R. F. and Pratt, J. W. (1984). The accuracy of peizer approximations to the hypergeometric distribution, with comparisons to some other approximations. Journal of the American Statistical Association, 79(385):49-60.

Liu, C. B. and Wang, H. L. (2014). Researches on the high order moments of hypergeometric distribution. Journal of Inner Mongolia Normal University (Natural Science Edition), 43:23-25. In Chinese.

Ma, C. S. (1999). Representations, bounds and approximations for tail probabilities of multivariate non-central hypergeometric and negative hypergeometric distributions. Journal of Statistical Computation and Simulation, 62(3):237258.

Mano, S. (2017). Partition structure and the a-hypergeometric distribution associated with the rational normal curve. Electronic Journal of Statistics, 11(2):4452-4487.

Molenaar, W. (1973). Simple approximations to poisson, binomial, and hypergeometric distributions. Biometrics, 29(2):403-407.

Ord, J. K. (1968). Approximations to distribution functions which are hypergeometric series. Biometrika, 55(1):243-248.

Panaretos, J. (1983). An elementary characterization of the multinomial and the multivariate hypergeometric distributions. Lecture Notes in Mathematics, 982:156-164.

Progri, I. F. (2011). Geolocation of RF Signals-Principles and Simulations. Springer, New York.

Progri, I. F. (2021a). Efficient computation of the generalized parabolic cylinder function distribution. Journal of Geolocation, Geo-information, and Geointelligence, 2021:13-29. Article ID 2021071602.

Progri, I. F. (2021b). Landmark computation of the generalized parabolic cylinder function distribution. Journal of Geolocation, Geo-information, and Geointelligence, 2021:30-44. Article ID 2021071603. 
Progri, I. F. (2021c). Special cases of the generalized parabolic cylinder function distribution. Journal of Geolocation, Geo-information, and Geo-intelligence, 2021:45-56. Article ID 2021071604.

Riordan, J. (1937). Moment recurrence relations for binomial, poisson and hypergeometric frequency distributions. Annals of Mathematical Statistics, $8: 103-111$.

Sibuya, M. and Shimizu, R. (1981). The generalized hypergeometric family of distributions. Annals of the Institute of Statistical Mathematics, 33(2):177190.

Skibinsky, M. (1970). A characterization of hypergeometric distributions. Journal of the American Statistical Association, 65(330):926-929.

Takayama, N., Kuriki, S., and Takemura, A. (2018). A-hypergeometric distributions and newton polytopes. Advances in Applied Mathematics, 99:109-133.

Themangani, R., Porwal, S., and Magesh, N. (2020). Generalized hypergeometric distribution and its applications on univalent functions. Journal of Inequalities and Applications, 2020(1).

Tohma, Y., Yamano, H., Ohba, M., and Jacoby, R. (1991). The estimation of parameters of the hypergeometric distribution and its application to the software-reliability growth-model. IEEE Transactions on Software Engineering, 17(5):483-489.

Tripathi, R. C. and Gurland, J. (1977). General family of discrete distributions with hypergeometric probabilities. Journal of the Royal Statistical Society Series B-Statistical Methodology, 39(3):349-356.

Wu, T. (1993). An accurate computation of the hypergeometric distribution function. ACM Transactions on Mathematical Software, 19(1):33-43.

Zhang, Y. Y., Rong, T. Z., and Li, M. M. (2019). Expectation identity for the binomial distribution and its application in the calculations of high-order bino- 
mial moments. Communications in Statistics-Theory and Methods, 48:5467-

5476. 